\title{
A Method for Reconstruction of the Neutral Particle Source Function in Helical Magnetically Confined Plasma
}

\author{
P.R. GONCHAROV, T. OZAKI, E.A. VESHCHEV ${ }^{1)}$ and S. SUDO \\ National Institute for Fusion Science, Toki 509-5292, Japan \\ ${ }^{1)}$ Graduate University for Advanced Studies, Hayama 240-0193, Japan
}

(Received 4 December 2006 / Accepted 26 March 2007)

\begin{abstract}
A numerical method is described that can be used to estimate the source of neutral particles within a helical plasma column as a function of the magnetic surface. The method is applicable to the data analysis of passive line-integral diagnostics of escaping neutrals. The magnetic surface structure taken from magnetohydrodynamic equilibrium calculations defines the kernel of the integral equation. A regularized solution is obtained over a discrete grid by minimizing the appropriate objective functional. The corresponding linear system is solved in terms of least squares using QR algorithm. The application is discussed to one particular charge-exchange diagnostic on the Large Helical Device and the model profile reconstruction examples are presented.
\end{abstract}

(C) 2007 The Japan Society of Plasma Science and Nuclear Fusion Research

Keywords: neutral particle diagnostic, line-integral measurement, helical plasma, non-circular isoline, regularization, ion distribution function

DOI: $10.1585 /$ pfr.2.S1074

\section{Introduction}

Multidirectional nonperturbing charge-exchange diagnostics based on high resolution atom energy spectrometers are used on the Large Helical Device (LHD) to study the ion component heating mechanisms and fast ion confinement by measuring the escaping neutral particle fluxes. The particle source $f(\rho)$ is not localized in contrast to the methods employing a diagnostic neutral particle beam or charge exchange on a solid pellet ablation cloud. Several possible approaches to the localization of such measurements on LHD were overviewed in [1] where a general description of the charge-exchange neutral particle diagnostic background, geometry of such experiments and the formulation of the measured quantity may be found. A new 20-channel analyzer for simultaneous measurements of the energy resolved neutral particle flux along twenty observation lines was described in [2].

This paper presents a numerical method realized in FORTRAN for the calculation of the neutral particle source function radial distribution $f(\rho)$ from lineintegrated passive measurement data. The basic integral relation between these quantities was discussed in [3] for LHD geometry along with the numerical simulation of the measured fluxes. The source function reconstruction algorithm requires the integral kernel calculation using the known geometry of measurements and the magnetohydrodynamic (MHD) equilibrium data, a certain treatment of the singularity at $\rho_{\mathrm{min}}$, and the application of linear regularization techniques.

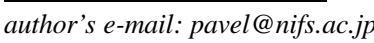

\section{Problem Formulation}

It is assumed that the neutral particle flux is measured from a thin viewing cone so that the source function integral over the observable plasma volume is reduced to the sight line integral. The diagnostic étendue factor will be omitted in the formulas herein. The attenuation of the atomic flux along the observation direction is supposed to be negligible. The relationship between the local source function and the measured line integral flux is given by the following Volterra integral equation of the first kind

$$
\int_{\rho}^{1} K(\rho, \xi) f(\xi) \mathrm{d} \xi=\Phi(\rho),
$$

where the kernel

$$
K(\rho, \xi)=Q^{+}(\rho, \xi)-Q^{-}(\rho, \xi)
$$

has two branches $Q^{+}(\rho, \xi)=\mathrm{d} \Lambda / \mathrm{d} \xi>0$ and $Q^{-}(\rho, \xi)=$ $\mathrm{d} \Lambda / \mathrm{d} \xi<0$ reflecting the integration variable change from the sight line distance $\Lambda$ to the effective radius $\xi$ along the two different monotonicity intervals explained in Fig. 1 (a) (negative branch from $\xi=1$ to $\xi=\rho$ and positive branch from $\xi=\rho$ to $\xi=1$ ). These two branches shown in Fig. 1 (b) are not necessarily symmetric and depend on the magnetic surface geometry. For circular concentric isoilnes of maximum radius $a$ formula (1) simplifies to the classical direct Abel transform and the analytical expression for the kernel becomes

$$
K(\rho, \xi)=2 a \xi / \sqrt{\xi^{2}-\rho^{2}} .
$$

The kernels of the form (2) typically have an integrable singularity at the lower integration limit. To reduce its in- 

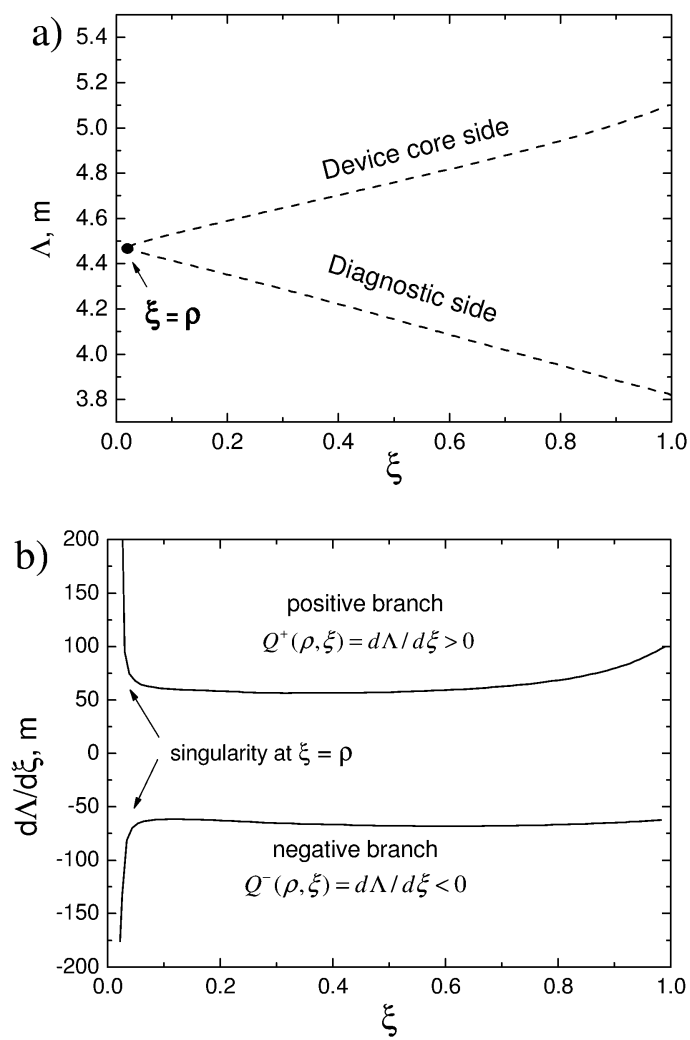

Fig. 1 (a) Two monotonicity intervals of the effective radius $\xi$ along the sight line distance $\Lambda$. (b) Positive and negative branches of the general Abel transform kernel for an arbitrary isoline shape.

fluence, one can perform a nonlinear change of the integration variable from $\xi$ to $t$ as follows

$$
t=\sqrt{\xi^{2}-\rho^{2}}
$$

Explicit formulas for the kernel in some particular cases of the magnetic surface shape can be found in [4]. For an arbitrary isoline shape it is practically comfortable to find analytical approximations of $Q^{+}(\rho, \xi)$ and $Q^{-}(\rho, \xi)$ functions for the diagnostic viewing direction using the magnetic surface structure data obtained from MHD equilibrium calculations for the particular plasma device.

Energy resolved fluxes are usually measured in experiments. It should be noted that $f(\rho)$ is then the source function of atoms in a certain energy range. Thus, a system of equations (1) for each energy channel rather than one equation should be solved to estimate the radially resolved energy spectrum.

\section{Solution Method}

Consider a set of $N$ chord integrated measurement results $\Phi_{j}$ and let $\rho_{j}$ be the minimum effective radius along the $j$ th chord, $j \in \overline{1, N}$. This determines a, generally speaking, non-uniform grid $\rho_{1}, \ldots, \rho_{N}$. The discretization of the problem (1) using the trapezoidal formula leads to a system of linear algebraic equations

$$
\mathrm{Kf}=\Phi
$$

with an upper triangular $N \times N$ matrix $K$ given by

$$
\mathrm{K}_{i j}=\left\{\begin{array}{l}
\kappa_{j} K\left(\rho_{i}, \rho_{j}\right), \quad j \geq i \\
0, \quad j<i
\end{array}\right.
$$

with $\left\{\begin{array}{l}\kappa_{1}=1 / 2\left(\rho_{2}-\rho_{1}\right) ; \quad \kappa_{N}=1 / 2\left(\rho_{N}-\rho_{N-1}\right) \\ \kappa_{j}=1 / 2\left(\rho_{j+1}-\rho_{j-1}\right), \quad j \in \overline{2, N-1}\end{array}\right.$

The exact solution of (4) corresponds to zero value of the 2 -norm of the residual vector

$$
R\{f\}=\|K \mathrm{f}-\Phi\|_{2} .
$$

Since the problem is known to be incorrectly posed, it is suitable to apply Tikhonov regularization method [5-7] to find the solution that minimizes the functional

$$
R_{\alpha}\{f\}=\|K \mathrm{f}-\Phi\|_{2}+\alpha\|D \mathrm{f}\|_{2} .
$$

The vector Df is the discrete counterpart of the integral

$$
\int_{0}^{1}\left|f^{\prime}(\xi)\right|^{2} d \xi \approx \frac{1}{2} \sum_{j=1}^{N}\left(\frac{\varphi_{j}}{\sqrt{\delta_{j}}}\right)^{2}
$$

The discretization is done again by the trapezoidal formula and the central difference derivatives contain

$$
\left\{\begin{array}{l}
\delta_{1}=\rho_{2}-\rho_{1}, \quad \varphi_{1}=\mathrm{f}_{2}-\mathrm{f}_{1} \\
\delta_{j}=\rho_{j+1}-\rho_{j-1}, \quad \varphi_{j}=\mathrm{f}_{j+1}-\mathrm{f}_{j-1}, \quad j \in \overline{2, N-1} \\
\delta_{N}=\rho_{N}-\rho_{N-1}, \quad \varphi_{N}=\mathrm{f}_{N}-\mathrm{f}_{N-1}
\end{array}\right.
$$

The right hand side of (8) has the form of the quadratic norm of a vector Df, where D is a tridiagonal matrix

$$
\begin{aligned}
& D_{1,1}=-1 / \sqrt{2 \delta_{1}} ; \quad D_{N, N}=1 / \sqrt{2 \delta_{N}} \\
& \left\{\begin{array}{l}
D_{i, i+1}=1 / \sqrt{2 \delta_{i}} \\
D_{i+1, i}=-1 / \sqrt{2 \delta_{i+1}}
\end{array}\right.
\end{aligned}
$$

for $i \in \overline{1, N-1}$.

The requirement of the minimization of the functional (7) is equivalent to the following overdetermined system of $2 N$ linear algebraic equations

$$
\left[\begin{array}{l}
K \\
\sqrt{\alpha} D
\end{array}\right] \mathrm{f}=\left[\begin{array}{l}
\Phi \\
0
\end{array}\right]
$$

which is to be solved in the least squares sense. Either QR algorithm or the system of normal equations can be used for this purpose. In the former case a decomposition of the $2 N \times N$ matrix of system (9) is calculated to represent it as a product of an orthogonal $2 N \times 2 N$ matrix $\mathrm{Q}$ and an upper triangular $2 N \times N$ matrix R. Since orthogonal transformations preserve the 2-norm, the least squares condition reduces to a readily soluble upper triangular system. In the latter case the least squares condition is re-written in the form of a symmetric $N \times N$ system solved by standard methods. The detailed description of these methods is given in the classical monograph [8]. 


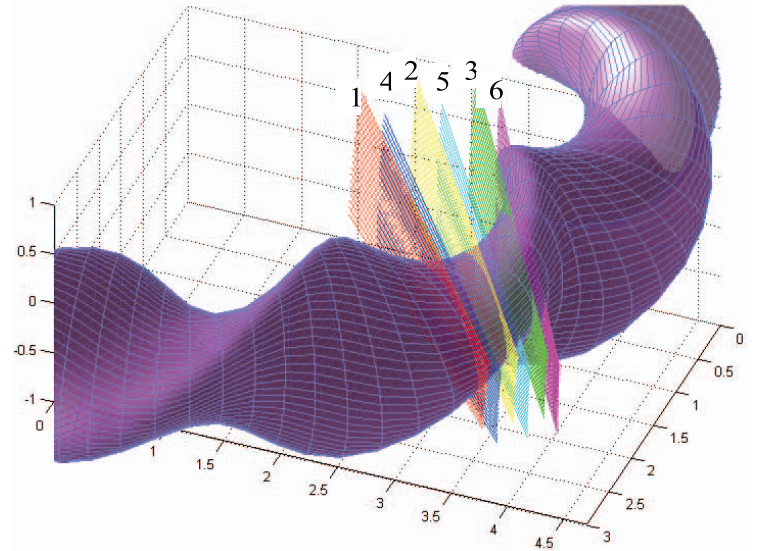

Fig. 2 Examples of the diagnostic cross-sections of the LHD plasma column available for the fan pattern scanning experiment with SDNPA [9].

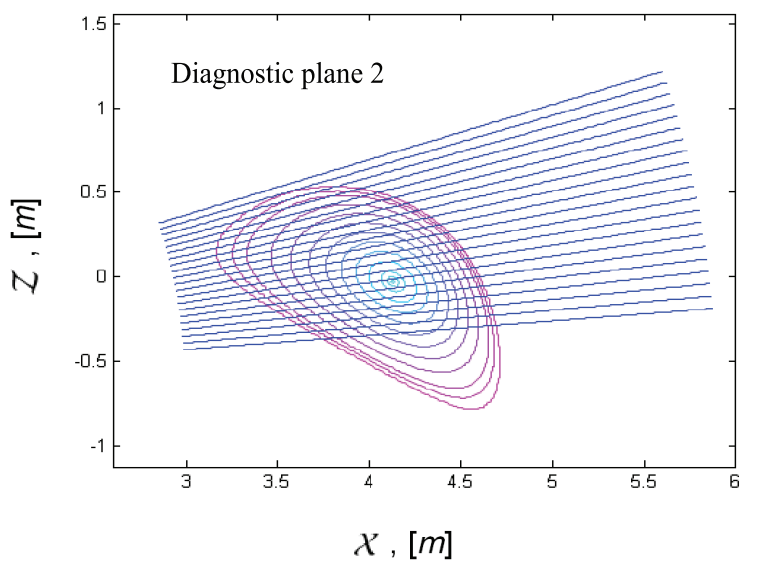

Fig. 3 Structure of the isolines and the sheaf of sight lines in the SDNPA diagnostic cross-section 2.

\section{Test Calculation Results}

The method has been tested assuming LHD magnetic surface geometry and the diagnostic sightlines corresponding to the Silicon Detector-based Neutral Particle Analyzer [9]. This diagnostic is capable of performing a scan of the plasma column in six vertical planes shown in Fig. 2. The vertical scan range, 20 sightline positions and the structure of the magnetic surfaces in the plane \#2 are shown in Fig. 3.

The isolines need to be a system of nested convex closed curves without self-intersections. These conditions are satisfied for this diagnostic plane. The innermost and the outermost values of the effective radius in this example are $\rho_{1}=0.024$ and $\rho_{20}=0.998$. Fig. 4 illustrates the data required for the integral kernel calculation. The measurable signal along these 20 sight lines has been simulated by the numerical evaluation of the integral (1) for three test profile shapes:
1). $f(\rho)=A \exp \left(-\rho^{2} / \sigma^{2}\right)$,
2). $f(\rho)=A(1-\rho)^{2}$,

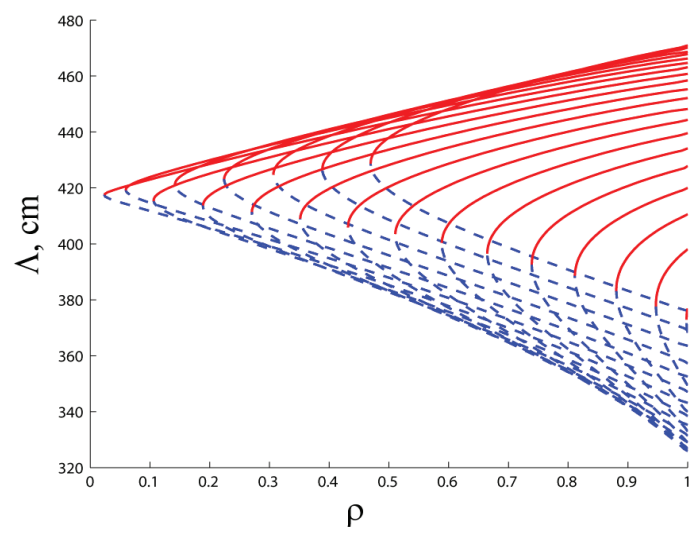

Fig. 4 Monotonicity intervals along the 20 sightlines in the SDNPA diagnostic cross-section 2.

Table 1 Residual vector characteristics.

\begin{tabular}{|c|c|c|c|}
\hline Case \# & $\left\|r_{\alpha}\right\|_{2} / \sqrt{N}$ & $\left|r_{\alpha}\right|_{\min }$ & $\left|r_{\alpha}\right|_{\max }$ \\
\hline 1 & 0.070 & $2.2 \times 10^{-3}$ & 0.147 \\
\hline 2 & 0.073 & $2.4 \times 10^{-4}$ & 0.203 \\
\hline 3 & 0.063 & $3.3 \times 10^{-4}$ & 0.121 \\
\hline
\end{tabular}

3). $f(\rho)=A\left(1-\rho^{2}\right)^{2}$.

The simulated sight line integral data was used as the input for the reconstruction method. Fig. 5 shows the obtained reconstructed profiles and the initial predefined source function curves. Table 1 summarizes the achieved values of the residual vector $r_{\alpha}=f-f^{*}$ between the vector $f$ of exact source values and the reconstructed vector $f^{*}$. The regularization parameter $\alpha$ values used in each case are shown on Fig. 5. The root-mean-square error values $\left\|r_{\alpha}\right\|_{2} / \sqrt{N}$ do not exceed $10 \%$ of the profile maxima in these examples. Maximum and minimum absolute deviations $\left|r_{\alpha}\right|_{\max }$ and $\left|r_{\alpha}\right|_{\text {min }}$ between the initial and the reconstructed profiles are also shown in the table. The solution smoothness and the accuracy of the obtained profile reconstruction depend on the regularization parameter choice. More sophisticated techniques exist [5] that enable automatic $\alpha$ adjustment.

\section{Summary}

A fast simple algorithm for local neutral particle source function reconstruction has been realized and tested for LHD geometry. The obtained regularized solutions are close to the predefined test profiles and the curve smoothness is achieved by choosing an appropriate regularization parameter. An automatic choice is preferable compared to the $a$ priori setting approach.

The method described here does not take into account the angular dependence of the neutral particle source function. This is often the case due to the anisotropy of the ion distribution function influenced by neutral beam injection or ion cyclotron radiofrequency heating. If the angle 

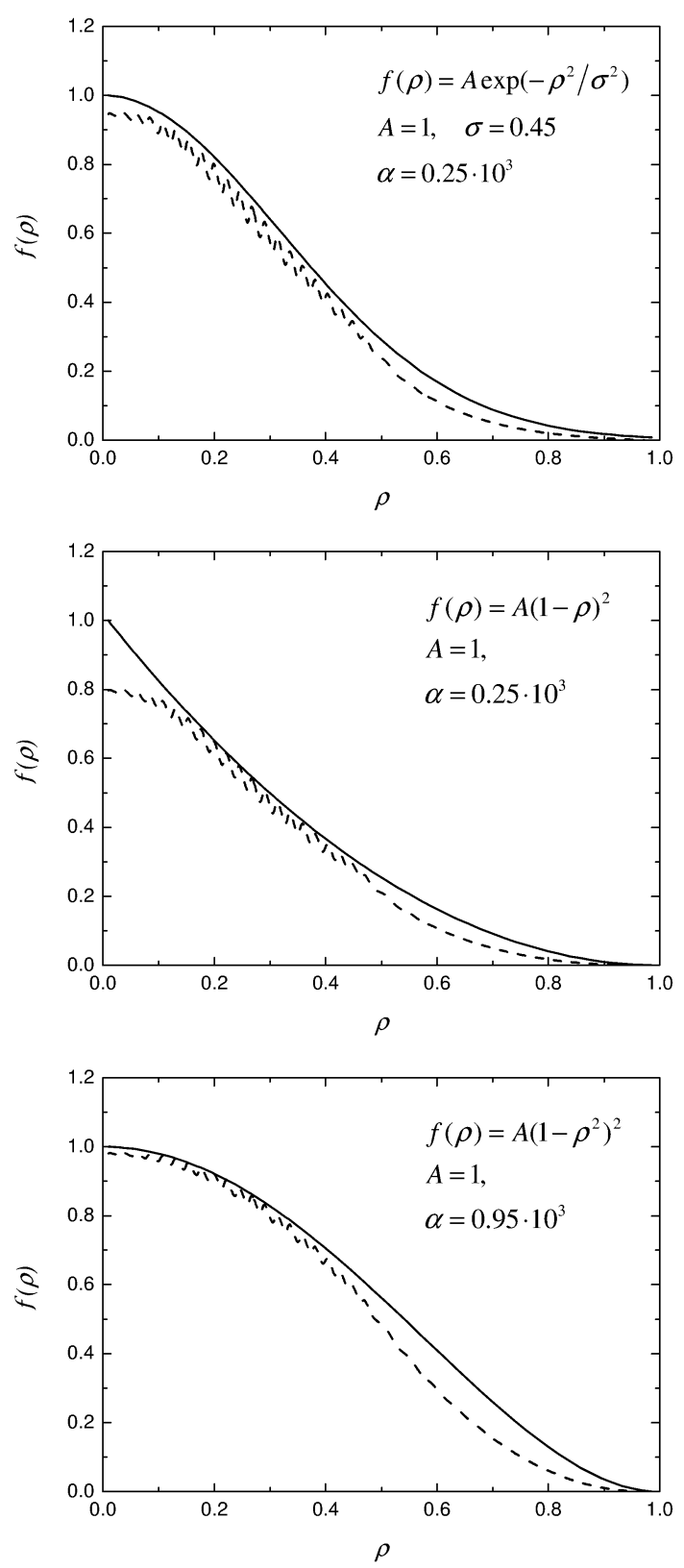

Fig. 5 Regularized solutions of the integral equation (dashed BSpline curves) and exact source functions (soild curves) for three different test profile shapes. between the diagnostic observation direction and the magnetic field varies significantly along the line of sight within the plasma, the local neutral particle source function reconstruction may become a much more complicated task. The angular distribution of suprathermal alpha particles produced in nuclear fusion reactions is, however, isotropic.

The presented algorithm is suitable not only in particle diagnostic data analysis, but for any kind of radiation source function reconstruction as well, assuming the source to be equal on a magnetic surface. The reconstructed radial dependence provides additional information for comparisons and cross-checks using the localized active diagnostic data.

[1] P.R. Goncharov, J.F. Lyon et al., J. Plasma Fusion Res. Series 6,314 (2004).

[2] E.A. Veshchev, T. Ozaki et al., Rev. Sci. Instrum. 77, 10F129 (2006).

[3] E.A. Veshchev, P.R. Goncharov et al., Rev. Sci. Instrum. 77, 10F116 (2006).

[4] V.V. Pickalov and T.S. Melnikova, Plasma Tomography (Nauka, Novosibirsk, 1995).

[5] A.N. Tikhonov and V.A. Arsenin, Solution of Ill-posed Problems (Winston \& Sons, Washington, 1977).

[6] V.A. Morozov, Methods for solving incorrectly posed problems (Springer-Verlag, Berlin, 1984).

[7] P.K. Lamm and L. Eldén, SIAM J. Numerical Analysis 34, 1432 (1997).

[8] G. Golub and C. Loan, Matrix Computations (The Johns Hopkins University Press, Baltimore, 1996).

[9] J.F. Lyon et al., Rev. Sci. Instrum. 74, 1873 (2003). 